\title{
Edición independiente y globalización editorial: el caso de los editores de ensayos «críticos» en Francia
}

\author{
Edition indépendante et globalisation: le cas des éditeurs d'essais \\ «critiques» en France
}

\author{
Sophie Noël \\ Universidad Paris 13 / \\ Labsic - CESSP \\ nolsophie@yahoo.fr
}

\begin{abstract}
Resumen
Este artículo trata sobre los editores de crítica social aparecidos en Francia a finales de la década del 80 , los cuales se autodefinen a partir de una identidad política específica. Sus editoriales encarnan una definición particular de independencia y, en el cruce entre una concepción económica, intelectual y política, permiten observar concretamente cómo es movilizado el término de independencia, objeto de luchas por su definición entre los diferentes actores de la cadena del libro. Luego de resituar el lugar de estos editores en el campo editorial francés, el texto interroga sobre los factores que permiten explicar su existencia en un contexto económico poco favorable. Se considera relevante, en este sentido, examinar en qué medida estas estructuras logran establecerse en los intersticios del sistema y cuáles son los límites que enfrentan.
\end{abstract}

Palabras Clave: Independencia, libros, edición, compromiso político.

\section{Résumé}

Cette présentation porte sur les éditeurs de critique sociale apparus à partir de la fin des années 1980 en France, qui se définissent par une identité politique. Ces maisons d'édition incarnent une définition particulière de l'indépendance, au carrefour d'une conception économique, intellectuelle et politique. Elles permettent d'observer de manière concrète comment est mobilisé le thème de l'indépendance, qui est l'objet de luttes de définition entre les différents acteurs de la chaîne du livre. Après avoir resitué ces éditeurs dans le champ éditorial français, on se posera la question des facteurs permettant d'expliquer leur existence dans un contexte économique peu favorable. L'intérêt est d'examiner dans quelle mesure 
ces structures parviennent à jouer dans les interstices du système, et quelles sont les limites quelles rencontrent.

Mots Clés: Indépendance, livres, édition, engagement politique.

El presente artículo abordará un sector específico de la edición independiente en Francia, a saber, los editores de "crítica social" o, en un sentido más amplio, de ensayo en ciencias sociales, que surgieron a fines de los años ochenta en el momento de la intensificación de la crítica al neoliberalismo y a la globalización.

Estas editoriales se definen bajo una identidad política globalmente de izquierda o "comprometida", que aparece como una ruptura o renovación, en un momento donde el campo editorial francés había, de cierta manera, abandonado los debates políticos e intelectuales que caracterizaron los años 60 y 70. Designaremos a estas editoriales, para no caer en una mera simplificación, como "críticas", ya que es interesante estudiar en qué medida éstas encarnan una definición particular de la independencia donde coinciden concepciones económicas e intelectuales pero también políticas, que las posicionan frente a ciertas derivas asociadas a la evolución del campo de la cultura en general. A este respecto, nos referimos a la defensa de la diversidad contra la "estandarización de la producción”, al rechazo de someterse a lógicas puramente "mercantiles" en nombre de la autonomía de la producción intelectual, y a la voluntad de concebir los libros como armas particularmente poderosas en la lucha por las ideas. Las editoriales estudiadas permiten observar de manera muy concreta cómo se movilizan actualmente ciertas temáticas (la independencia, el pluralismo), a menudo imprecisas y mal definidas, que son objeto de fuertes "luchas de definición" entre los diferentes actores de la cadena del libro, particularmente en el campo de la "producción restringida". Ellas ilustran de manera ejemplar las ambigüedades del término "independiente" (Robin, 2008) que es objeto de apropiaciones que compiten entre sí.

En esta dirección, primeramente vamos a resituar a estas pequeñas editoriales en el campo editorial francés antes de plantearnos la pregunta de cuáles son los factores que permiten explicar su existencia en un contexto económico difícil -pero al mismo tiempo muy favorable si se le compara, por ejemplo, con aquel en el que se inscriben los editores chilenos-, como ha sido expuesto a lo largo de las dos jornadas de este Coloquio. Nuestro interés consiste en examinar en qué medida estas estructuras logran jugar en los intersticios del sistema para crearse una posición relativamente

1 Pierre Bourdieu establece, al interior del campo cultural, una distinción entre un polo de producción restringida y otro de la gran producción. El primero produce bienes simbólicos en un ciclo productivo lento, que están destinados a un público reducido, y son objeto de una evaluación entre los pares. El segundo produce bienes destinados a un consumo rápido e indiferenciado (estandarizado) cuya principal instancia de evaluación es el mercado (Bourdieu, 1992). 
duradera, así como explicar cuáles son los límites con los que estas mismas se encuentran.

\section{EDITORES «HETERODOXOS»}

La edición ha experimentado profundas transformaciones, en Francia y en el mundo entero, a partir de los años 80: olas de fusiones de sellos, internacionalización y reforzamiento de las lógicas financieras. El campo editorial actual presenta una estructura profundamente asimétrica, que ve coexistir dos grupos dominantes (Hachette y Editis) que engloban un gran número de prestigiosas casas editoriales ${ }^{2}$, un puñado de editores de talla mediana con tendencia a fortalecerse gracias a las operaciones de fusión (Gallimard-Flammarion, La Martinière-Le Seuil) y una inmensa cantidad de estructuras más o menos artesanales -más de 3000 según el INSEE $^{3}$ - que conforman el "oligopolio con franjas" (Reynaud, 1982) ${ }^{4}$.

Este fenómeno no es históricamente nuevo. Como lo han mostrado los historiadores del libro (Mollier, 1988; Chartier y Martin, 1990), la edición ha experimentado importantes movimientos de concentración y de racionalización industrial desde el siglo XIX, y se ha caracterizado siempre por la existencia de un número importante de pequeñas estructuras efímeras en mayor o menor medida. No obstante, a partir de los años 60 este movimiento se ha intensificado, lo cual nos lleva a interrogarnos acerca de la capacidad de resistencia de estas pequeñas estructuras mal equipadas para afrontar una lógica de mercado cada vez más “dura con los débiles”. De hecho, los pequeños editores (vistos como un solo sector) se encuentran confrontados a actores que compiten no sólo apoyándose en poderosas herramientas de difusión y distribución, sino que además controlan en gran parte los puntos de venta. Se trata entonces de una competencia que de esta manera maneja el conjunto de la cadena del libro, desde la concepción a la venta, sin mencionar sus vínculos particulares con los medios de comunicación ${ }^{5}$, que no serán abordados aquí.

A la luz de este contexto económico particular, podemos preguntarnos por la existencia en Francia de un movimiento significativo de creación de pequeñas editoriales independientes, en un momento en que sus probabilidades de sobrevivencia no han hecho más que degradarse a partir de los años 80 . En efecto, cerca

\footnotetext{
2 Hachette realiza una cifra de negocios de 2038 millones de euros, casi tres veces superior a la de Editis (con 706 millones de euros) que le sigue inmediatamente en la competencia.

3 El Instituto Nacional de Estadísticas.

4 Ver artículo Concentración, independencia y diversidad editorial (25-31), publicado en este número.

5 El grupo Hachette es dueño de un conjunto de medios de comunicación: prensa escrita, radio, kioskos en los aeropuertos, estaciones de tren y hospitales (Relay), etc.
} 
de la mitad de las editoriales fundadas entre 1980 y 2005 no han logrado mantenerse más de cinco años, mientras que el balance de creación/desaparición de editoriales se ha convertido en negativo a partir de 1998 (Legendre y Abensour, 2007).

Sin embargo, si nos concentramos en el sub-sector muy particular de los editores de crítica social, no podemos sino constatar un movimiento de creación relativamente sostenido a fines de la década de los 80 , que toma vuelo en los 90 , sin detenerse desde entonces: una cincuentena de editoriales que podemos definir de identidad "crítica" o "heterodoxa" han aparecido en los últimos 25 años, con diferentes suertes (Noël, 2012). ¿Cómo caracterizarlas? Se trata de estructuras heterogéneas cuya producción (que va de dos a cuarenta títulos por año) representa una gota en el océano de la producción editorial francesa. Algunas son muy marginales, mientras que otras han adquirido cierta notoriedad, como por ejemplo Raisons d'agir, Agone, Les Prairies ordinaires, o Amsterdam. Sus fondos editoriales varían desde una pequeña decena de títulos a varios centenares en el caso de las más antiguas y sus cifras de negocios oscilan entre los 15000 euros a los cerca de 500000 euros. Respecto a sus miembros estables, éstos varían de una a seis personas, en su mayoría trabajando sin salario fijo, como voluntarios, y viven gracias a trabajos realizados en paralelo.

Cerca de la mitad de estas editoriales hacen su distribución de manera profesional, principalmente a través de empresas medianas como Harmonia Mundi y Les Belles Lettres. Sólo una pequeñísima minoría se autodifunde o lo hace a través de estructuras alternativas como Court-Circuit, que se encarga principalmente de la difusión de asociaciones políticamente comprometidas ${ }^{6}$. En consecuencia, estamos hablando de una nebulosa que reagrupa asociaciones relativamente marginales pero también de algunas empresas que se han ido profesionalizando. Su producción es muy diversa y comprende desde testimonios hasta textos de análisis e investigaciones especializadas, publica panfletos, encuestas y también reedita textos políticos "clásicos" (clásicos del marxismo o libertarios). Muchas editoriales se han desarrollado a partir de una ambiciosa política de traducciones, con el fin de hacer accesible en francés los textos mayores de las ciencias sociales que no habían sido traducidos nunca ni del inglés, ni de otras lenguas más "periféricas" (italiano, alemán, castellano ... ). Este es el caso, por ejemplo, de la editorial de L'Eclat, que publicó en 1989 “Tiré à part”, una colección de filosofía analítica a partir de traducciones de autores como John Searle, Karl-Otto Apel y Hilary Putman. Podemos citar igualmente las ediciones Amsterdam, fundadas en 2005, que han desarrollado una política sistemática de traducción de textos que emanan de los Estudios de género (Judith Butler), de los Estudios subalternos como también de los Estudios post-coloniales (Marcus Rediker).

6 Court-circuit es una estructura de difusión-distribución alternativa creada en 2004 por el editor de corte libertario Nautilus, que reúne cerca de cuarenta editores también de tendencia "libertaria". (N. del T. : Dejó de funcionar en el 2013). 
A pesar de su fuerte carácter político, estas editoriales se caracterizan por un distanciamiento dela política tradicional, prefiriendo el calificativo de "comprometido" o de "activista" al de militante, el cual perciben como una descalificación. Habría que describir en detalle sus modos de posicionamiento y sus trayectorias como editores, cosa que excede las posibilidades de este artículo. Solamente precisaremos que estos editores son representativos de dispositivos de compromiso de fuerte inclinación intelectual, emergiendo a fines de los años 80 y sobre todo en los 90, en un contexto social de renovación contestataria (Agrikoliansky y Sommier, 2005) sobre la forma bastante vaga de una "resistencia al neoliberalismo" en la esfera de influencia, por ejemplo, del movimiento alter mundialista ATTAC.

\section{¿UN MODELO ALTERNATIVO SOBRE EL MERCADO GLOBAL?}

Pasaremos a evaluar los recursos de los que disponen estos editores con el fin de procurar instalarse de manera durable en el campo editorial francés, en un ámbito conocido como difícil: el de las ciencias sociales (Barluet, 2005). Diversos factores, de variada importancia, pueden ser movilizados para explicar esta dinámica de creación. En primer lugar, la edición es tradicionalmente una actividad que posee una débil barrera de ingreso (Mauger, 2007): un capital mínimo, una habitación en un departamento, un computador, un programa de edición, algunas ideas y un poco de audacia "bastan" para crear una estructura editorial. La mayor parte de los editores evocados aquí comenzaron de esta manera "haciendo un poco de todo", en una posición de editor frágil. Esta situación se produce debido al número limitado de puestos disponibles en el sector de la edición, lo cual sumado a la sobreproducción de egresados, ha contribuido a reforzar esta tendencia.

Por su parte, las innovaciones tecnológicas han agudizado aún más esta facilidad, abriendo nuevas posibilidades en materia de marketing y de venta directa. Internet ofrece en efecto una vitrina para nada costosa para llegar a lectores potenciales, sin necesidad de intermediarios. Michel Valensi, responsable de Éditions de L'Éclat, fue el primero en poner a disposición algunos de sus títulos para descarga gratuita desde su sitio, lo que tuvo una repercusión positiva sobre las ventas de sus libros en librería. Muchos editores realizan más que el $20 \%$ de sus ventas a través de su sitio Internet, ya sea en formato electrónico o en papel. Esta solución financieramente ventajosa -por la ausencia de descuentos y de gastos de difusión, como de devolucionespresenta por su parte el inconveniente mayor de eludir a los libreros. Como lo ha señalado Anne-Marie Métailié ${ }^{7}$ en su intervención, los libreros constituyen desde su

7 Ver artículo Las funciones del editor independiente en el campo editorial (169-176), publicado 
función un apoyo esencial para los editores independientes, debido a la gran cercanía de sus posiciones e intereses. En Francia, unos 200 a 300 libreros cumplen un rol indispensable, difundiendo y recomendando libros, frente a una enorme producción editorial (cerca de 79000 títulos - novedades y reimpresiones- fueron publicados en 2010 según el Sindicato Nacional de la Edición $)^{8}$, compensando de algún modo la escasez de acceso a los medios que tienen estos editores. En términos globales, los libreros fuera de los grandes hipermercados especializados, representan el 45\% del mercado minorista en Francia a pesar de la sostenida erosión de su posición (SNE, 2011).

Junto con las librerías llamadas "de calidad", que ponen permanentemente en valor los fondos de los editores, y más específicamente la producción de las editoriales independientes, organizando encuentros con los autores y otros eventos, los editores críticos pueden apoyarse en las redes asociativas o militantes, en función de sus temáticas de preferencia. Es el caso específico de una editorial como Syllepse, cuyas colecciones, en particular aquellas que tratan sobre el tema del mundo del trabajo, son motivadas por sindicatos u organizaciones como la Fundación Copernic ${ }^{9}$. Estos canales alternativos pueden revelarse como espacios eficaces, produciendo aportes económicos apreciables en el caso de los libros de preventa. Igualmente, varios salones del libro de carácter militante o especializado (el Salón del libro libertario, el Salón de los editores independientes o especialmente el Pequeño salón del libro político) han aparecido a su vez a partir de la última década, creando espacios de expresión y de exposición valorizados por estas editoriales y sus autores, como son los debates y encuentros.

Resulta difícil estudiar las condiciones y las posibilidades de estas pequeñas editoriales en el campo actual, sin mencionar las ayudas públicas a las cuales estas pueden aspirar en nombre de una demanda de corrección de los desequilibrios del mercado por parte del Estado. Estas ayudas constituyen un aporte nada despreciable para la caja de las estructuras caracterizadas por una debilidad de sus recursos financieros y un difícil acceso a los préstamos bancarios. Existen distintos niveles institucionales que ponen recursos a disposición: las municipalidades, los departamentos, las regiones (nivel que reúne a los departamentos), como también la Dirección Regional de Asuntos Culturales (DRAC).

Mencionaremos el caso de Ediciones Les Prairies ordinaires, que en 2006 pudieron

\footnotetext{
en este número.

8 El Sindicato National de la Edición, SNE, realiza desde 1954 un estudio estadístico anual sobre la edición, por encargo del Ministerio de la Industria.

9 Un círculo de reflexión crítica creado en 1998, con el fin de "devolver a su lugar aquello que el liberalismo ha hecho funcionar al revés".
} 
lanzar una ambiciosa colección de traducciones de ensayos en ciencias sociales, llamada "Penser/Croiser", gracias al Consejo regional d' Ile-de-France, que financió la mitad del proyecto. A nivel nacional, un interlocutor central es el Centro Nacional del libro (CNL), organismo dependiente del Ministerio de cultura, cuyo presupuesto se nutre principalmente de un impuesto a la reprografía. Este último ofrece apoyos a la traducción, préstamos a taza cero, ayudas para el desarrollo de colecciones, para el equipamiento, etc. La mayoría de los editores críticos han recurrido a estas ayudas, aunque tengan tendencia a relativizar el patrocinio público, que no concuerda bien con sus reivindicaciones de independencia. Sin embargo, los menos profesionalizados entre ellos tienden a autoexcluirse y no postulan a las ayudas públicas, en la medida en que no podrán responder a los criterios solicitados (por ejemplo contar con un traductor profesional y pagarle una tarifa mínima). A grandes rasgos, las estructuras editoriales generalistas ${ }^{10}$ medianas son las principales beneficiarias de estas subvenciones, aún cuando los pequeños editores independientes están lejos de estar ausentes (Cartellier, 2008).

En definitiva, el mantenimiento de una reserva dinámica de pequeños editores sobre este segmento específico de producción no puede explicarse únicamente por elementos estructurales o institucionales. Un factor importante se debe al hecho de que el oficio de editor cuenta todavía en Francia con un destacado prestigio social, especialmente en lo que respecta a su dimensión de "intelectual comprometido", que refleja una tradición vigente aún hoy en día (Casanova, 1999). Los editores críticos se refieren a menudo de esta manera a las "grandes figuras" de la profesión como François Maspero, fundador en 1959 de la editorial homónima, quien construyó un catálogo central para la difusión de las ideas de izquierda en el período 1960-1970 (Hage, 2009); o Jérôme Lindon, director de Éditions de Minuit, editorial fundada durante la ocupación alemana durante la Segunda guerra mundial en Francia (Simonin, 1994), también es percibido como una figura emblemática de la edición "exigente" y sin concesiones ${ }^{11}$. Los beneficios de distinción simbólica asociados a la actividad de editor, reforzados por su dimensión independiente, no pueden ser subestimados, especialmente al interior de un sector con un perfil altamente intelectual como lo es el de las ciencias humanas. Este perfil ejerce una fuerte atracción sobre una población mayoritariamente masculina, que proviene principalmente de clases medias o

10 En Francia, la "edición generalista" corresponde a los editores de literatura y de ensayo, los cuales se sitúan frente a los editoreses pecializados en un sector específico. Generalmente son editores prestigiosos como Gallimard, Le Seuil o Albin Michel, cuyos catálogos reúnen particularmente ensayos de ciencias humanas de un amplio espectro (historia, sociología, filosofía...) dirigido a un público "culto".

11 Jérôme Lindon fue uno de los defensores más activos de la Ley sobre el precio único para el libro, en vigencia desde 1981. 
superiores, que posee diplomas académicos, y que busca posiciones profesionales en un contexto económico difícil (Chauvel, 2006).

El desarrollo de un discurso de resistencia frente a la globalización editorial y frente a un tipo de administración enceguecida por las cifras de gestión en la profesión, contribuye de hecho a vigorizar estas gratificaciones simbólicas. Uno de sus ejes es la acentuación de un saber-hacer ${ }^{12}$ o experticia artesanal, que permite a los editores deslindarse de las "grandes máquinas sin alma" de la edición globalizada, en las cuales los libros serán banalizados y desatendidos de los cuidados que requieren. Una editora parisina, que creó su editorial en los años 90, lo expresa con claridad:

"Existe una cosa que yo quisiera subrayar también, y es que aquí se trabaja de una manera que puede ser arcaica, más artesanal y seria que en las grandes editoriales. Esto es lo que yo les escucho decir a los autores cuando pasan por nuestra editorial" (Entrevista, noviembre 2006)

\section{LA AMBIVALENCIA FRENTE AL DESARROLLO}

El ciclo económico particularmentelargo del libro, que posee un tiempo de retorno de los rendimientos de muy largo plazo (a diferencia de los gastos, que son inmediatos) sumado al carácter fundamentalmente incierto de esta actividad (Caves, 2000), ocasiona fuertes dificultades de liquidez para los pequeños editores, lo que viene a alimentar la concentración del sector. La dependencia frente a las instituciones crediticias -las veces que éstas aceptan financiarles - o de los organismos públicos, que de manera corriente pueden no volver a renovarles las subvenciones acordadas, se torna problemático.

Muchos de los editores entrevistados se encuentran en situaciones económicas delicadas, con un ritmo de producción que ha bajado desde hace algunos años. Otros, como Éric Hazan, fundador de la editorial La Fabrique, consideran que sólo le deben su sobrevivencia a un rechazo a toda forma de desarrollo que involucre una dependencia financiera: "a partir del momento en que necesitas dinero para desarrollarte y que te lo prestan, estás muerto”. Permanecer al margen aparece como el único medio de escape a las presiones del mercado, ya que un aumento en el volumen de producción implica contratar personal, endeudarse, etc. En consecuencia, la producción se limita a una decena de títulos por año, con salarios de media jornada. Este tipo de posición contribuye a hacer más borrosa la frontera entre una práctica

12 N. del T.: del francés "savoir faire" (similar al términoinglés know-how) que alude al conjunto de conocimientos, experiencias, competencias y capacidad de resolver problemas prácticos, acumulados a lo largo del ejercicio de una actividad. 
amateur y una profesional (Poliak, 2006), lo que va a representar una dimensión en juego ${ }^{13}$ crucial, como lo ha demostrado claramente Bertrand Legendre, planteando la pregunta de la inscripción a largo plazo de estas estructuras en el campo editorial. Los pequeños editores independientes están de hecho obligados a afirmar su identidad sobre dos frentes distintos: uno, que los ubica de manera opuesta a los grandes editores, frente a quienes deben probar su "suplemento de alma", y el otro que los separa de los amateurs, que no efectúan un "verdadero trabajo editorial". Se trata de una posición incómoda, que les exige permanentes redefiniciones y una exaltación de sus competencias específicas. El dispositivo o conjunto de elementos que acompañan los textos (las notas, comentarios y prefacios) es por lo general bastante cuidado, acorde con la concepción exigente del rol de editor que quieren encarnar. Estos esfuerzos se inscriben al interior de una forma de ethos artesanal que se manifiesta en el amor por el "objeto bello" y en el orgullo del trabajo bien hecho, no desprovisto de un cierto romanticismo. El tipógrafo constituye de esta manera una figura de fuerte identificación que alude a un ideal de dominio de la cadena del libro en su conjunto, lo cual data de los primeros años de la edición.

Otro fenómeno que se ha ido extendiendo son las "recuperaciones" de algunas iniciativas editoriales desarrolladas por estos editores críticos, particularmente en sus expresiones académico-militantes. Portadores de una innegable innovación editorial a partir de los años 90, tanto en lo que respecta a la forma, con pequeñas obras de intervención a precio accesible, como fueron L'Esprit frappeur o Raisons d'agir, como sobre el fondo, gracias a temáticas en boga con los "nuevos movimientos sociales” (Sommier, 2003), estas iniciativas encontraron rápidamente un eco en los editores generalistas. Estos últimos han invertido todo su savoir-faire y su capacidad logística en colecciones que exploran temáticas o formatos parecidos-especialmente en torno a la crítica al capitalismo- entrando a competir fuertemente con las editoriales críticas en su propio terreno. El caso de la publicación de textos de la asociación ATTAC es profundamente revelador de este fenómeno: después de haberle confiado sus primeros títulos a Syllepse, un pequeño editor alternativo políticamente cercano, se prefirió entregar su producción a Mille et Une Nuits ${ }^{14}$, que estaba en condiciones de asegurar mayor difusión y mejores condiciones. A través de este ejemplo podemos presenciar un clásico circuito de difusión de la innovación desde la periferia hacia el centro, que es particularmente cruel en la situación mencionada, considerando sobretodo la disimetría de los recursos respectivos.

Confrontados a estas múltiples dificultades, estos pequeños editores se ven

13 N. del T.: la autora utiliza la palabra "enjeu" que traducimos al español como "lo que está en juego".

14 Editorial perteneciente al Grupo Hachette. 
obligados a "hacer de la necesidad virtud", como lo explicara Pierre Bourdieu, limitando sus costos fijos de manera radical. De esta manera, sólo los más profesionales recurren a la subcontratación, mientras que la mayoría de los editores tienen que trabajar de manera voluntaria durante varios años, incluso a veces permanentemente, demostrando una gran polivalencia. Deben efectuar trabajos en paralelo para mantenerse, normalmente ligados a la edición, como el packaging ${ }^{15} \mathrm{o}$ el diseño. Las tareas de corrección tipográfica, edición y traducción son a menudo realizadas por ellos mismos o por allegados, de manera gratuita, incluso gracias a diversas combinaciones (trabajos de ayuda ${ }^{16}$, pasantías o prácticas profesionales) que les permite mantener unos gastos fijos muy bajos. Esta forma de economía paralela o de "economía de favores" (Thompson, 2010), caracterizada por la precariedad y el ascetismo, permite a la mayor parte de estos editores no perder dinero, a falta de poder ganarlo, con los límites que esto claramente implica en cuanto a las posibilidades de profesionalización de la actividad.

De manera característica la autoexplotación es percibida por algunos como una elección, una prueba de abnegación propia al esquema del arte. Ella se compensa por los beneficios simbólicos asociados a un estatus de intelectual comprometido y a la "pasión" inherente al oficio (Moati, 1992). Como lo señala un empleado de una estructura de difusión alternativa: "no sabemos lo que son las horas extras (pagadas) y el derecho laboral lo hemos tirado a la basura”. Asimismo, el vocabulario de la pasión y de la vocación aparece permanentemente en los discursos y en la manera en que los editores se presentan a sí mismos, lo cual hace posible una valorización de una actividad desconectada de los beneficios económicos, que tiene como razón principal los beneficios intelectuales (Freidson, 1986). El tema de su autoexplotación, y la de los otros (pasantes, colaboradores freelance, etc.), constituye a menudo un punto ciego para estos editores, más aún cuando muestran una tendencia a ocultar que esto contradice los propios principios éticos que ellos defienden y que les empuja hacia las prácticas de las "grandes" estructuras, en cuya oposición se definen.

Como se lamenta un editor parisino, cuya estructura pasó a ser filial de La Martinière en 2005:

"La independencia de los pequeños editores oculta una realidad miserable, la explotación de sí mismo y de los otros, las negociaciones a fin de mes con los bancos, los proveedores y los autores, a los cuales no se les ha pagado a tiempo".

15 Esto se refiere a un tipo de servicio que opera bajo la modalidad de entrega de un "producto terminado", haciéndose cargo de las distintas etapas de producción (la fotografía, la gráfica, la edición, etc). Son estructuras que se han especializado (desarrollando esta experticia particular) en la elaboración de textos de venta fácil como manuales de cocina, jardinería, etc.

16 El Estado subvenciona indirectamente ciertos puestos de trabajo, reduciendo las cargas sociales que debe pagar la empresa. 
Esta plena conciencia de las presiones económicas, que no pueden ignorar sin correr el riesgo de desaparecer, puede conducir a algunos de ellos al agotamiento y a la tentación de delegar el ámbito de la gestión a una estructura mayor, con el objeto de poder concentrarse en los contenidos políticos e intelectuales del trabajo editorial (la selección de obras, el trabajo con los autores, la construcción del catálogo, etc.). De hecho, su tamaño no les permite separar principio de gestión y principio artístico, como sucede normalmente en las industrias culturales (Chiapello, 1998). Esta constatación nos lleva a la doble función del intermediario cultural, a la vez artista y comerciante (Bourdieu, 1992). Por su parte, el equilibrio entre estas dos dimensiones fundamentales del oficio de editor es por definición precario, sobre todo si se considera que pone en relación principios a priori difícilmente conciliables, como son la prudencia económica y la audacia intelectual (Bourdieu, 1999).

Esta rápida presentación del caso de los editores independientes de crítica social en Francia ilustra el carácter paradójico del actual mercado de la edición, donde coexisten grandes grupos que siguen creciendo permanentemente, con pequeñas editoriales, situadas en la periferia del campo editorial, que se van reduciendo de más en más. Estas últimas se encuentran reducidas a ocupar un rol económico insignificante, desde la perspectiva de los actores dominantes, pero encarnando, al mismo tiempo, ciertos valores esenciales (el pluralismo, la independencia ... ) para el funcionamiento del campo editorial en su vertiente simbólica. De esta manera, contribuyen a crear una creencia colectiva necesaria para el buen funcionamiento del campo editorial en su conjunto.

En efecto, existen dos maneras de interpretar la permanencia de un número relativamente estable de pequeñas estructuras editoriales frente a los grandes grupos. Podemos considerar que ellas son la señal de una sana resistencia de ciertos nichos de creación frente a un movimiento de homogeneización y de racionalización del sector, resistencia que mantiene cueste lo que cueste la vertiente artesanal del oficio de editor (Benghozi y Moire, 1986), el cual es el más propicio a la creación y a la conservación de una cierta diversidad editorial. O podemos, a la inversa, observar que la evolución es completamente favorable a los grandes grupos, dejando a las pequeñas estructuras el espacio de poder jugar un rol de "laboratorio profesional colectivo", invirtiéndose en la generación de proyectos y de autores desconocidos (pero con alguna posibilidad de transformarse en un "nombre"), con alta probabilidad de, ya sea absorberlos en el futuro, o de recuperar sus catálogos ${ }^{17}$ (Rouet, 2007). Sin duda, cada una de estas dos tesis es parcialmente correcta. Nos queda por agregar que hemos constatado que los editores críticos han sabido adaptarse e inventar, a su nivel, nuevos modos

17 Lo que se muestra con la absorción de Climats por Flammarion en 2005, o la adquisición por parte de Actes Sud, en 2009, del 65\% del capital de Textuel. 
de producción viables, apoyándose en un discurso político de resistencia eficaz que les asegura una cierta legitimidad en el espacio público, pero pagando un precio de una alta y creciente autoexplotación. Al igual que en el caso de otras industrias culturales como la música o el cine, que han vivido esquemas de evolución similares, la experiencia pareciera indicar que habrá siempre un espacio para editores "valientes" y "dinámicos" situados en el margen, mientras la edición pueda beneficiarse de un contexto institucional relativamente protector y de aquello que podríamos calificar como un ejército de reserva de editores aspirantes, dispuestos a invertir su trabajo sin escatimar esfuerzos.

\section{Referencias bibliográficas}

Agrikoliansky, E. y Sommier, I. (Dir.). (2005). Radiographie du mouvement altermondialiste. Paris: la Dispute.

Barluet, S. (2004). Edition de sciences humaines et sociales: le cour en danger. Paris: PUF.

Benghozi, J. P. y Moire, C. (1986). L’Opéra de quat'sous: productions souterraines et productions culturelles. En Rouet, F. (Dir.), Économie \& culture, Industries culturelles (Vol.3) (pp. 237-243). Paris: La Documentation française.

Bourdieu, P. (1992). Les règles de l’art. Paris: Le Seuil.

Bourdieu, P. (1999). Une révolution conservatrice dans l'édition. Actes de la recherche en sciences sociales, 126-127, 3-27.

Caves, R. (2000). Creative industries: Contracts between art and commerce. Cambridge: Harvard University Press.

Casanova, P. (1999). La République mondiale des lettres. Paris: Le Seuil.

Chartier, R. y Martin, H. J. (Dir.). (1983-1986), Histoire de l'édition française. Paris : Promodis.

Chauvel, L. (2006). Les classes moyennes à la dérive. Paris: Le Seuil.

Chiapello, E. (1998). Artistes versus managers. Paris: Métailié.

Freidson, E. (1986). Les professions artistiques comme défi à l'analyse sociologique. Revue française de sociologie, 27, 431-443.

Hage, J. (2009). Une brève histoire des librairies et des éditions Maspero. En Collectif, François Maspero et les paysages humains. Lyon: La Fosse aux ours/À plus d'un titre.

Legendre, B. y Abensour, C. (2007). Regards sur l'édition, 2, Les nouveaux éditeurs (1988-2005). Paris: La Documentation française.

Mauger, G. (2007). Droits d'entrée. Modalités et conditions d'accès aux univers artistiques. Paris: Maison des sciences de l'homme.

Martin, H. J. y Febvre, L. (1958). Lapparition du livre. Paris: Albin Michel. 
Moati, P. (1992). La filière du roman : de la passion à la rationalité marchande. Cahiers de l'économie du livre, 7, 103-137.

Mollier, J. Y. (1988). Largent et les Lettres : histoire du capitalisme d'édition, 18801920. Paris: Fayard.

Noël, S. (2012). Lédition indépendante critique : engagements politiques et intellectuels. Villeurbanne: Presses de l'Enssib.

Poliak, C. (2006). Aux frontières du champ littéraire. Sociologie des écrivains amateurs. Paris: Economica.

Reynaud, B. (1999). L'emprise des groupes sur l'édition française au début des années 1980. Actes de la recherche en sciences sociales, 130, 3-10.

Robin, C. (2008). La notion d'indépendance éditoriale. Communication \& langages, $156,53-62$.

Rouet, F. (2007). Le livre. Mutations d'une industrie culturelle. Paris: La Documentation française.

Simonin, A. (1994). Les éditions de Minuit, 1942-1955, le devoir d'insoumission. Paris: IMEC.

Sommier, I. (2003). Le renouveau des mouvements contestataires à l'heure de la mondialisation. Paris: Flammarion.

Syndicat national de l'édition. (2011). Repères statistiques 2010.

Thompson, J. B. (2010). Merchants of Culture: the Publishing Business in the 21st Century. Cambridge: Polity Press. 